\title{
Group Sequential Tests with Outcome-Dependent Treatment Assignment
}

\author{
Christopher Jennison \\ Department of Mathematical Sciences, \\ University of Bath, Bath BA2 7AY, U. K. \\ and \\ Bruce W. Turnbull \\ School of Operations Research and Industrial Engineering \\ Cornell University, Ithaca, New York 14853-3801, U. S. A.
}

\begin{abstract}
We consider group sequential comparisons of two treatments in which subjects' treatment assignments may be based on previously observed responses. We present theory for the joint distribution of the sequence of test statistics in such a study and show that this has a standard form when treatment assignment is based only on the current estimate of the treatment difference. This theory supports the inclusion of adaptive sampling rules in standard group designs, maintaining the usual Type I and II error probabilities. Simulation results show the expected number of subjects receiving the inferior treatment can be reduced by up to 10 or $15 \%$ with increases in total sample size of around 1 to $3 \%$. More aggressive strategies can reduce the number on the inferior treatment by up to 30 or $40 \%$, on average, but at the price of substantial increases in total sample size.
\end{abstract}

Key words: Adaptive sampling rule, Clinical trial, Data dependent allocation, Error spending, Group sequential test, Inferior treatment number, Two-armed bandit.

\section{Introduction}

When two treatments are compared in a clinical trial, the accumulating data will often indicate that one treatment is likely to be superior well before there is sufficient evidence to terminate the trial with a firm conclusion. Weighting patient randomization towards the treatment with more favorable responses at an interim stage can help reduce the number of subjects allocated to the inferior treatment over the course of the study. We 
shall refer to the expected number of subjects receiving the inferior treatment as the "inferior treatment number" (ITN) and the expected total sample size as the "average sample number" (ASN). Reduction of the ITN is usually accompanied by an increase in ASN. If, as is often the case, the total sample size is closely related to the time when results can be reported and a superior treatment made available to the wider population, interest should focus on treatment allocation rules which reduce ITN with only a small increase in ASN.

Historically, the idea of basing the assignment of subjects to treatment on current estimates of the treatment difference dates back at least to Thompson (1933). Robbins (1952) formulated the "two-armed bandit" problem in which the aim is to maximize the number of successfully treated cases when allocating a sequence of subjects to two treatments with unknown and unequal success probabilities. Anscombe (1963) and Colton (1963) proposed a "horizon" formulation in which the total number of subjects to be treated is fixed and the results of a comparison in the first phase determine the choice of treatment for all remaining subjects in the second phase. Many authors have continued to develop research in this area: the book by Berry \& Fristedt (1985) surveys work on bandit problems and selected references and several recent review articles are cited in Jennison \& Turnbull (2000, Sec. 17.4). Despite the large volume of research, this methodology has had little impact on clinical trial practice. Indeed, the relevance of proposed adaptive sampling strategies to the needs of medical research has been seriously questioned by some discussants - see, for example, Peto's (1985) remarks on the papers by Bather (1985) and Armitage (1985). Interest in the area has been rekindled by the use of response-adaptive designs in the ECMO trials reported by Cornell, Landenberger \& Bartlett (1986) and Ware (1989) and in two trials of anti-depression drugs sponsored by Eli Lilly and Co. (Tamura et al., 1994; Andersen, 1996), the latter being an interesting example of a fourarmed adaptive trial. It is now standard practice to monitor data regularly during the course of a medical trial and for the study protocol to specify a group sequential stopping rule. In this paper, we shall show how adaptive treatment allocation can be incorporated into commonly used group sequential procedures.

The use of adaptive allocation rules in a continuously monitored sequential probability ratio test (SPRT) has been described by Robbins \& Siegmund (1974), Hayre (1979) and Hayre \& Turnbull (1981a). In this case, simplifications arise from the definition of the SPRT in terms of a likelihood ratio and the lack of overshoot of the stopping boundary which allows use of martingale results to calculate expected sample sizes. More general distribution theory is required to support the construction of group sequential tests. We present this general theory for a simple two-treatment comparison in Section 2.1, explaining the relation to Robbins \& Siegmund's results; this theory was stated without a complete proof by Jennison \& Turnbull (2000, Sec. 17.3). In Section 2.2 we extend the theory to the group sequential analysis of normal linear models, including the case of a two-treatment comparison with adjustment for covariates. Jennison \& Turnbull (2000, Sec. 17.2) define adaptive allocation procedures based on this theory and demonstrate 
the reductions achieved in ITNs. We analyze a subset of these results in Section 3 and note the benefits and limitations of this approach. We conclude in Section 4 with further discussion of practical issues.

\section{Distribution Theory}

\subsection{A Simple Two-Treatment Comparison}

Suppose we wish to compare two treatments, $A$ and $B$, from which independent observations $X_{A i} \sim N\left(\mu_{A}, \sigma^{2}\right)$ and $X_{B i} \sim N\left(\mu_{B}, \sigma^{2}\right), i=1,2, \ldots$, are available. We shall assume throughout this paper that the variance $\sigma^{2}$ is known. Data are collected in stages and we denote by $n_{A k}$ and $n_{B k}$ the cumulative numbers of observations collected

on treatments $A$ and $B$ respectively by the end of stage $k=1, \ldots, K$. The successive differences $n_{A k}-n_{A, k-1}$ and $n_{B k}-n_{B, k-1}$ are the group sizes on the two treatment arms. Define the estimates

$$
\widehat{\mu}_{A k}=\frac{1}{n_{A k}} \sum_{i=1}^{n_{A k}} X_{A i} \quad \text { and } \quad \widehat{\mu}_{B k}=\frac{1}{n_{B k}} \sum_{i=1}^{n_{B k}} X_{B i}, \quad k=1, \ldots, K,
$$

and associated information levels

$$
\mathcal{I}_{A k}=\frac{n_{A k}}{\sigma^{2}} \quad \text { and } \quad \mathcal{I}_{B k}=\frac{n_{B k}}{\sigma^{2}}, \quad k=1, \ldots, K
$$

It is convenient to work in terms of the scores

$$
S_{A k}=\widehat{\mu}_{A k} \mathcal{I}_{A k}=\frac{1}{\sigma^{2}} \sum_{i=1}^{n_{A k}} X_{A i}
$$

and

$$
S_{B k}=\widehat{\mu}_{B k} \mathcal{I}_{B k}=\frac{1}{\sigma^{2}} \sum_{i=1}^{n_{B k}} X_{B i} .
$$

The parameter of interest is

$$
\theta=\mu_{A}-\mu_{B}
$$

and at analysis $k$ we estimate this by

$$
\widehat{\theta}_{k}=\widehat{\mu}_{A k}-\widehat{\mu}_{B k}=\frac{S_{A k}}{\mathcal{I}_{A k}}-\frac{S_{B k}}{\mathcal{I}_{B k}} .
$$

The information for $\theta$ at analysis $k$ is

$$
\mathcal{I}_{\theta k}=\left\{\operatorname{Var}\left(\hat{\theta}_{k}\right)\right\}^{-1}=\frac{\mathcal{I}_{A k} \mathcal{I}_{B k}}{\mathcal{I}_{A k}+\mathcal{I}_{B k}}
$$


and the score statistic for $\theta$ is

$$
S_{\theta k}=\widehat{\theta}_{k} \mathcal{I}_{\theta k}=\frac{S_{A k} \mathcal{I}_{B k}-S_{B k} \mathcal{I}_{A k}}{\mathcal{I}_{A k}+\mathcal{I}_{B k}}
$$

Defining

$$
W_{k}=S_{A k}+S_{B k},
$$

the statistics $S_{\theta k}$ and $W_{k}$ are jointly sufficient for $\mu_{A}$ and $\mu_{B}$ at analysis $k$.

If the values of the group sizes are pre-specified, the sequences $\left\{S_{A k} ; k=1, \ldots, K\right\}$, $\left\{S_{B k} ; k=1, \ldots, K\right\}$ and $\left\{S_{\theta k} ; k=1, \ldots, K\right\}$ are distributed as Brownian motions with drifts $\mu_{A}, \mu_{B}$ and $\theta$ observed at times $\left\{\mathcal{I}_{A k} ; k=1, \ldots, K\right\},\left\{\mathcal{I}_{B k} ; k=1, \ldots, K\right\}$ and $\left\{\mathcal{I}_{\theta k} ; k=1, \ldots, K\right\}$, respectively. If group sizes are random but independent of previous parameter estimates, $\widehat{\mu}_{A k}, \widehat{\mu}_{B k}$ and $\widehat{\theta}_{k}$, this remains true conditionally, given the observed group sizes. Our objective is to establish conditions under which the $S_{\theta k}$ sequence retains its Brownian motion distribution when future group sizes are allowed to depend on previously observed parameter estimates.

\section{The Sampling Procedure}

We suppose initial group sizes, $n_{A 1}$ and $n_{B 1}$, are fixed. At each analysis $k=1, \ldots, K-1$ we allow the adaptive sampling rule to give the next pair of group sizes, $n_{A, k+1}-n_{A k}$ and $n_{B, k+1}-n_{B k}$, as functions of $S_{\theta k}$ and the previous group sizes. Equivalently, the group sizes are functions of $S_{\theta k}$ and $G_{k}$ where $G_{k}=\left\{n_{A 1}, \ldots, n_{A k}, n_{B 1}, \ldots, n_{B k}\right\}$. Note that $G_{k}$ contains sufficient information to determine $\mathcal{I}_{A j}, \mathcal{I}_{B j}$ and $\mathcal{I}_{\theta j}$ for all $j \leq k$ and $S_{\theta k}$

and $G_{k}$ together determine $\hat{\theta}_{k}$. Since it is rarely possible to attain target sample sizes exactly, we shall also allow the possibility of random group sizes, letting the distribution of $n_{A, k+1}-n_{A k}$ and $n_{B, k+1}-n_{B k}$ depend on $S_{\theta k}$ and $G_{k}$. Typically, target sample sizes will be set as functions of $S_{\theta k}$ and $G_{k}$ and the numbers of observations actually recorded will vary around these values.

The following results remain valid if the sampling rule at stage $k$ is also allowed to depend on all previous estimates $\hat{\theta}_{j}, j=1, \ldots, k$, although such rules are not of great

interest since $\widehat{\theta}_{k}$ and $\mathcal{I}_{\theta k}$ are sufficient for $\theta$ at this point. However, we shall see later that the stage $k$ sampling rule should not be allowed to depend on $W_{k-1}$, the information about $\mu_{A}$ and $\mu_{B}$ orthogonal to $S_{\theta, k-1}$.

\section{Theorem 1.}

Under the forms of adaptive sampling described above,

$$
S_{\theta 1} \mid \mathcal{I}_{\theta 1} \sim N\left(\mathcal{I}_{\theta 1} \theta, \mathcal{I}_{\theta 1}\right)
$$

and for each $k=2, \ldots, K$,

$$
S_{\theta k}-S_{\theta, k-1} \mid \mathcal{I}_{\theta 1}, \ldots, \mathcal{I}_{\theta k}, S_{\theta 1}, \ldots, S_{\theta, k-1} \sim N\left(\left\{\mathcal{I}_{\theta k}-\mathcal{I}_{\theta, k-1}\right\} \theta, \mathcal{I}_{\theta k}-\mathcal{I}_{\theta, k-1}\right)
$$


Note that each information level $\mathcal{I}_{\theta k}$ can, and in general will, depend on the previous score statistics $S_{\theta 1}, \ldots, S_{\theta, k-1}$. The theorem states that each increment $S_{\theta k}-S_{\theta, k-1}$ is conditionally independent of $S_{\theta 1}, \ldots, S_{\theta, k-1}$ given $\mathcal{I}_{\theta 1}, \ldots, \mathcal{I}_{\theta k}$ : hence, the process $\left\{S_{\theta k} ; k=1, \ldots, K\right\}$ evolves as a Brownian motion observed at times $\left\{\mathcal{I}_{\theta k} ; k=1, \ldots, K\right\}$ but with the additional feature that the values $\mathcal{I}_{\theta k}$ depend on previously observed $S_{\theta j}$ s.

\section{Proof of Theorem 1.}

The proof is by induction with the inductive hypothesis

$$
\mathcal{H}_{k}:\left\{\begin{array}{l}
S_{\theta k}-S_{\theta, k-1} \perp S_{\theta 1}, \ldots, S_{\theta, k-1} \mid G_{k}, \\
S_{\theta k}-S_{\theta, k-1} \mid G_{k} \sim N\left(\left\{\mathcal{I}_{\theta k}-\mathcal{I}_{\theta, k-1}\right\} \theta, \mathcal{I}_{\theta k}-\mathcal{I}_{\theta, k-1}\right), \\
W_{k} \perp S_{\theta 1}, \ldots, S_{\theta k} \mid G_{k}, \\
W_{k} \mid G_{k} \sim N\left(\mathcal{I}_{A k} \mu_{A}+\mathcal{I}_{B k} \mu_{B}, \mathcal{I}_{A k}+\mathcal{I}_{B k}\right) .
\end{array}\right.
$$

Here the symbol $\perp$ denotes conditional independence and $S_{\theta, 0}$ and $\mathcal{I}_{\theta, 0}$ are both defined to be zero. The theorem follows directly from $\mathcal{H}_{1}, \ldots, \mathcal{H}_{K}$.

The initial hypothesis $\mathcal{H}_{1}$ is easily checked. For $k=1, \ldots, K-1$, we prove $\mathcal{H}_{k+1}$ from $\mathcal{H}_{k}$ by establishing the following results:

1. $S_{\theta, k+1}-S_{\theta k} \perp S_{\theta 1}, \ldots, S_{\theta k} \mid G_{k+1}$ and

$$
S_{\theta, k+1}-S_{\theta k} \mid G_{k+1} \sim N\left(\left\{\mathcal{I}_{\theta k}-\mathcal{I}_{\theta, k-1}\right\} \theta, \mathcal{I}_{\theta, k+1}-\mathcal{I}_{\theta k}\right),
$$

2. $W_{k+1} \perp S_{\theta 1}, \ldots, S_{\theta k} \mid G_{k+1}$ and

$$
W_{k+1} \mid G_{k+1} \sim N\left(\mathcal{I}_{A, k+1} \mu_{A}+\mathcal{I}_{B, k+1} \mu_{B}, \mathcal{I}_{A, k+1}+\mathcal{I}_{B, k+1}\right),
$$

3. $W_{k+1} \perp S_{\theta, k+1}-S_{\theta k} \mid G_{k+1}, S_{\theta 1}, \ldots, S_{\theta k}$.

Note that Results 2 and 3 imply $W_{k+1} \perp S_{\theta 1}, \ldots, S_{\theta, k+1} \mid G_{k+1}$ as needed for $\mathcal{H}_{k+1}$. In proving these results, we shall use extensions of the properties of $W_{k}$ stated in $\mathcal{H}_{k}$. Since $G_{k+1}$ comprises $G_{k}$ plus $n_{A, k+1}$ and $n_{B, k+1}$ and the distribution of $n_{A, k+1}$ and $n_{B, k+1}$ depends only on $G_{k}$ and $S_{\theta 1}, \ldots, S_{\theta k}$, it follows from $\mathcal{H}_{k}$ that also

$$
W_{k} \perp S_{\theta 1}, \ldots, S_{\theta k} \mid G_{k+1}
$$

and the conditional distribution of $W_{k}$ given $G_{k+1}$ is the same as that given $G_{k}$, i.e.,

$$
W_{k} \mid G_{k+1} \sim N\left(\mathcal{I}_{A k} \mu_{A}+\mathcal{I}_{B k} \mu_{B}, \mathcal{I}_{A k}+\mathcal{I}_{B k}\right) .
$$




\section{Result 1.}

A little algebra shows that $S_{\theta, k+1}-S_{\theta k}$ can be decomposed as

$$
\begin{gathered}
S_{\theta, k+1}-S_{\theta k}=\frac{\mathcal{I}_{B, k+1}}{\mathcal{I}_{A, k+1}+\mathcal{I}_{B, k+1}}\left(S_{A, k+1}-S_{A k}\right)-\frac{\mathcal{I}_{A, k+1}}{\mathcal{I}_{A, k+1}+\mathcal{I}_{B, k+1}}\left(S_{B, k+1}-S_{B k}\right) \\
\quad+\frac{\mathcal{I}_{A k} \mathcal{I}_{B, k+1}-\mathcal{I}_{B k} \mathcal{I}_{A, k+1}}{\left(\mathcal{I}_{A k}+\mathcal{I}_{B k}\right)\left(\mathcal{I}_{A, k+1}+\mathcal{I}_{B, k+1}\right)}\left(S_{A k}+S_{B k}\right) \\
=\frac{n_{B, k+1}}{n_{A, k+1}+n_{B, k+1}} \frac{1}{\sigma^{2}} \sum_{i=n_{A k}+1}^{n_{A, k+1}} X_{A i}-\frac{n_{A, k+1}}{n_{A, k+1}+n_{B, k+1}} \frac{1}{\sigma^{2}} \sum_{i=n_{B k}+1}^{n_{B, k+1}} X_{B i} \\
\quad+\frac{n_{A k} n_{B, k+1}-n_{B k} n_{A, k+1}}{\left(n_{A k}+n_{B k}\right)\left(n_{A, k+1}+n_{B, k+1}\right)} W_{k} .
\end{gathered}
$$

Given $G_{k+1}$, the first two terms in (4) taken together are normal with mean

$$
\frac{\mathcal{I}_{B, k+1}}{\mathcal{I}_{A, k+1}+\mathcal{I}_{B, k+1}}\left(\mathcal{I}_{A, k+1}-\mathcal{I}_{A k}\right) \mu_{A}-\frac{\mathcal{I}_{A, k+1}}{\mathcal{I}_{A, k+1}+\mathcal{I}_{B, k+1}}\left(\mathcal{I}_{B, k+1}-\mathcal{I}_{B k}\right) \mu_{B}
$$

and variance

$$
\frac{\mathcal{I}_{B, k+1}^{2}}{\left(\mathcal{I}_{A, k+1}+\mathcal{I}_{B, k+1}\right)^{2}}\left(\mathcal{I}_{A, k+1}-\mathcal{I}_{A k}\right)+\frac{\mathcal{I}_{A, k+1}^{2}}{\left(\mathcal{I}_{A, k+1}+\mathcal{I}_{B, k+1}\right)^{2}}\left(\mathcal{I}_{B, k+1}-\mathcal{I}_{B k}\right)
$$

Since these terms depend only on the data gathered in stage $k+1$, they are conditionally independent, given $G_{k+1}$, of $S_{\theta 1}, \ldots, S_{\theta k}$ and of the remaining term in (4). By (1) and (2), the third term in (4) is conditionally independent of $S_{\theta 1}, \ldots, S_{\theta k}$ given $G_{k+1}$ and its conditional distribution term given $G_{k+1}$ is normal with mean

$$
\frac{\mathcal{I}_{A k} \mathcal{I}_{B, k+1}-\mathcal{I}_{B k} \mathcal{I}_{A, k+1}}{\left(\mathcal{I}_{A k}+\mathcal{I}_{B k}\right)\left(\mathcal{I}_{A, k+1}+\mathcal{I}_{B, k+1}\right)}\left(\mathcal{I}_{A k} \mu_{A}+\mathcal{I}_{B k} \mu_{B}\right)
$$

and variance

$$
\frac{\left(\mathcal{I}_{A k} \mathcal{I}_{B, k+1}-\mathcal{I}_{B k} \mathcal{I}_{A, k+1}\right)^{2}}{\left(\mathcal{I}_{A k}+\mathcal{I}_{B k}\right)^{2}\left(\mathcal{I}_{A, k+1}+\mathcal{I}_{B, k+1}\right)^{2}}\left(\mathcal{I}_{A k}+\mathcal{I}_{B k}\right)
$$

Combining these results and simplifying the expressions for mean and variance gives our desired Result 1.

The routine but somewhat lengthy algebra required in this last step can be circumvented. If we consider a non-adaptive study with the same $\mathcal{I}_{A k}, \mathcal{I}_{A, k+1}, \mathcal{I}_{B k}$ and 
$\mathcal{I}_{B, k+1}$, it is easy to show directly that the mean and variance of $S_{\theta, k+1}-S_{\theta k}$ are as stated in Result 1. The mean and variance of $S_{\theta, k+1}-S_{\theta k}$ in this non-adaptive case can also be calculated through the decomposition described above and both methods will, of course, give the same answer. The algebra in this second calculation coincides with that for the adaptive case which must, therefore, also lead to Result 1. This line of reasoning may be applied elsewhere in the proofs of Theorems 1 and 2 although, for simplicity of exposition, we shall present direct arguments. However, the idea is important and shows that the key steps in the proofs are those establishing conditional independence to justify certain calculations, rather than the detailed computations themselves - whose results are familiar from the non-adaptive case.

Result 2.

We treat $W_{k+1}$ as the sum of $W_{k}$ and $W_{k+1}-W_{k}$. Now,

$$
\begin{aligned}
W_{k+1}-W_{k} & =\left(S_{A, k+1}-S_{A k}\right)+\left(S_{B, k+1}-S_{B k}\right) \\
& =\frac{1}{\sigma^{2}} \sum_{i=n_{A k}+1}^{n_{A, k+1}} X_{A i}+\frac{1}{\sigma^{2}} \sum_{i=n_{B k}+1}^{n_{B, k+1}} X_{B i} .
\end{aligned}
$$

Since $W_{k+1}-W_{k}$ only involves data gathered in stage $k+1$,

$$
W_{k+1}-W_{k} \perp S_{\theta 1}, \ldots, S_{\theta k} \mid G_{k+1}
$$

and with (1) this implies

$$
W_{k+1} \perp S_{\theta 1}, \ldots, S_{\theta k} \mid G_{k+1} .
$$

Furthermore,

$$
\begin{gathered}
W_{k+1}-W_{k} \mid G_{k+1} \sim \\
N\left(\left\{\mathcal{I}_{A, k+1}-\mathcal{I}_{A k}\right\} \mu_{A}+\left\{\mathcal{I}_{B, k+1}-\mathcal{I}_{B k}\right\} \mu_{B},\left\{\mathcal{I}_{A, k+1}-\mathcal{I}_{A k}\right\}+\left\{\mathcal{I}_{B, k+1}-\mathcal{I}_{B k}\right\}\right)
\end{gathered}
$$

and

$$
W_{k+1}-W_{k} \perp W_{k} \mid G_{k+1} .
$$

Combining (2), (5) and (6) completes our Result 2.

\section{Result 3.}

We can write $W_{k+1}$ as

$$
W_{k+1}=\left(W_{k+1}-W_{k}\right)+W_{k}=\left(S_{A, k+1}-S_{A k}\right)+\left(S_{B, k+1}-S_{B k}\right)+W_{k}
$$


and, by (3),

$$
\begin{gathered}
S_{\theta, k+1}-S_{\theta k}=\frac{\mathcal{I}_{B, k+1}}{\mathcal{I}_{A, k+1}+} \mathcal{I}_{B, k+1}\left(S_{A, k+1}-S_{A k}\right)-\frac{\mathcal{I}_{A, k+1}}{\mathcal{I}_{A, k+1}+\mathcal{I}_{B, k+1}}\left(S_{B, k+1}-S_{B k}\right) \\
+\frac{\mathcal{I}_{A k} \mathcal{I}_{B, k+1}-\mathcal{I}_{B k} \mathcal{I}_{A, k+1}}{\left(\mathcal{I}_{A k}+\mathcal{I}_{B k}\right)\left(\mathcal{I}_{A, k+1}+\mathcal{I}_{B, k+1}\right)} W_{k}
\end{gathered}
$$

Both these expressions involve new data from stage $k+1$ in $S_{A, k+1}-S_{A k}$ and $S_{B, k+1}-S_{B k}$ as well as the sum $W_{k}$ which is known at stage $k$. Conditionally on $S_{\theta 1}, \ldots, S_{\theta k}$ and $G_{k+1}$, $S_{A, k+1}-S_{A k}, S_{B, k+1}-S_{B k}$ and $W_{k}$ are independent with

$$
\begin{aligned}
& S_{A, k+1}-S_{A k} \sim N\left(\left\{\mathcal{I}_{A, k+1}-\mathcal{I}_{A k}\right\} \mu_{A}, \mathcal{I}_{A, k+1}-\mathcal{I}_{A k}\right) \\
& S_{B, k+1}-S_{B k} \sim N\left(\left\{\mathcal{I}_{B, k+1}-\mathcal{I}_{B k}\right\} \mu_{B}, \mathcal{I}_{B, k+1}-\mathcal{I}_{B k}\right)
\end{aligned}
$$

and, by $\mathcal{H}_{k}$

$$
W_{k} \sim N\left(\mathcal{I}_{A k} \mu_{A}+\mathcal{I}_{B k} \mu_{B}, \mathcal{I}_{A k}+\mathcal{I}_{B k}\right) .
$$

Thus, conditionally, $W_{k+1}$ and $S_{\theta, k+1}-S_{\theta k}$ are bivariate normal with covariance

$$
\begin{gathered}
\frac{\mathcal{I}_{B, k+1}}{\mathcal{I}_{A, k+1}+\mathcal{I}_{B, k+1}}\left(\mathcal{I}_{A, k+1}-\mathcal{I}_{A k}\right)-\frac{\mathcal{I}_{A, k+1}}{\mathcal{I}_{A, k+1}+\mathcal{I}_{B, k+1}}\left(\mathcal{I}_{B, k+1}-\mathcal{I}_{B k}\right) \\
+\frac{\mathcal{I}_{A k} \mathcal{I}_{B, k+1}-\mathcal{I}_{B k} \mathcal{I}_{A, k+1}}{\left(\mathcal{I}_{A k}+\mathcal{I}_{B k}\right)\left(\mathcal{I}_{A, k+1}+\mathcal{I}_{B, k+1}\right)}\left(\mathcal{I}_{A k}+\mathcal{I}_{B k}\right)=0
\end{gathered}
$$

and, therefore,

$$
W_{k+1} \perp S_{\theta, k+1}-S_{\theta k} \mid G_{k+1}, S_{\theta 1}, \ldots, S_{\theta k}
$$

as required for our Result 3 . This completes the proof.

Robbins \& Siegmund (1974) derived their SPRT without the full result of Theorem 1. By transforming the data to $X_{A i}-X_{A 1}, i=2,3, \ldots$, and $X_{B i}-X_{A 1}, i=1,2, \ldots$, they obtained variables whose distribution depends only on $\mu_{A}-\mu_{B}$ and used the usual likelihood ratio argument to establish error probabilities of the SPRT. Since their sampling rule must be a function of the transformed variables, it can depend on the full-data estimates $\widehat{\mu}_{A}$ and $\widehat{\mu}_{B}$ at any stage only through their difference $\widehat{\mu}_{A}-\widehat{\mu}_{B}$. Robbins \& Siegmund used the decomposition (4) in proving that the score process $\left\{S_{\theta k}\right\}$ can be embedded in a Brownian motion under a pre-specified sequence of observations. However, they only required a martingale property for the score process under adaptive sampling in their discussion of the expected sample sizes on each treatment arm. For group sequential tests, which cannot make use of the SPRT's likelihood ratio argument, the additional properties of normality and conditionally independent increments proved in Theorem 1 are required. 
Examination of a part of the proof of Theorem 1 helps explain how a sampling rule in which stage $k$ allocations are allowed to depend on $W_{k}$ can bias a sequential test under particular treatment means $\mu_{A}=\mu_{A}^{*}$ and $\mu_{B}=\mu_{B}^{*}$, say. Suppose at stage $k$ we examine both $S_{k}$ and $W_{k}$ and deduce that $\widehat{\mu}_{A k}>\mu_{A}^{*}$ and $\widehat{\mu}_{B k} \approx \mu_{B}^{*}$. If, indeed, $\mu_{A}=\mu_{A}^{*}$ and $\mu_{B}=\mu_{B}^{*}$, the effect of the high value of $\widehat{\mu}_{A k}$ on $\widehat{\mu}_{A, k+1}$ is progressively diluted as the sample size on treatment $A$ in stage $k+1$ increases, thus, a sampling rule which allocates a smaller proportion of observations to treatment $A$ in stage $k+1$ in response to a high value of $\widehat{\mu}_{A k}$ and a lower proportion in response to a low value of $\widehat{\mu}_{A k}$ will bias $\widehat{\theta}_{k+1}=\widehat{\mu}_{A, k+1}-\widehat{\mu}_{B, k+1}$ upwards. More generally, a rule which makes the coefficient of $W_{k}$ in (4) positive when $W_{k}$ exceeds its expectation and negative otherwise will cause an upwards bias in $\widehat{\theta}_{k+1}$. If, instead, we are told the value of $S_{k}$ but not $W_{k}$, we cannot tell whether the value of $S_{k}$ arises from $\widehat{\mu}_{A k}>\mu_{A}^{*}$ and $\widehat{\mu}_{B k} \approx \mu_{B}^{*}$ or, say, $\widehat{\mu}_{A k} \approx \mu_{A}^{*}$ and $\widehat{\mu}_{B k}<\mu_{B}^{*}$. In the latter situation, allocating fewer observations to treatment $A$ in stage $k+1$ would now bias $\widehat{\theta}_{k+1}$ downwards. Result 1 of the theorem shows that if sampling is based only on $S_{k}$, the potential biases in $\hat{\theta}_{k+1}$ of any sampling strategy cancel out when averaged over the conditional distribution of $\widehat{\mu}_{A k}$ and $\widehat{\mu}_{B k}$ given $S_{k}$.

\subsection{General Normal Linear Models}

We now consider the case where observations follow a normal linear model with a $p$-dimensional parameter vector $\boldsymbol{\beta}$ and the primary research question concerns the linear combination of parameters $\boldsymbol{c}^{T} \boldsymbol{\beta}$. An important special case is a two-treatment comparison with adjustment for covariates. There, $\beta_{1}$ represents the treatment effect under investigation and $\beta_{2}, \ldots, \beta_{p}$ are coefficients of covariate terms, and we set $c_{1}=1$ and $c_{2}=\ldots=c_{p}=0$.

Suppose observations $X_{1}, X_{2}, \ldots$ are collected group sequentially in up to $K$ groups and let $n_{k}$ be the total numbers of observations available at analysis $k, k=1, \ldots, K$. We suppose that, given covariate values $d_{i 1}, \ldots, d_{i p}$, the $i$ th observation is distributed as

$$
X_{i} \sim N\left(\sum_{i=1}^{p} d_{i j} \beta_{j}, \sigma^{2}\right) .
$$

Under adaptive sampling, the number of observations in a group may depend on previously observed data. Also the covariates associated with these observations depend on previous data and so the $X_{i}, i=1,2, \ldots$, are not formally independent. Our assumption is that for each $k=2, \ldots, K$, observations in group $k$ are conditionally independent of those in groups 1 to $k-1$ given their covariate values. The distributional result in Theorem 2 below can also be extended to correlated data, as might arise in a longitudinal study with repeated measurements on each subject. For simplicity, we prove the theorem in the uncorrelated case and explain its generalization at the end of this section.

Let $\boldsymbol{X}_{k}$ denote the vector of all $n_{k}$ observations available at analysis $k$. Thus, each $\boldsymbol{X}_{k}$ contains the elements of $\boldsymbol{X}_{k-1}$ plus the additional observations recorded in stage $k$. Let 
$\boldsymbol{D}_{k}$ be the $n_{k} \times p$ design matrix for $\boldsymbol{X}_{k}$ with $i$ th row consisting of $d_{i 1}, \ldots, d_{i p}$ and suppose $\boldsymbol{D}_{1}$, and therefore all later $\boldsymbol{D}_{k} \mathrm{~s}$, have rank $p$. In the absence of adaptive sampling we would write

$$
\boldsymbol{X}_{k} \sim \boldsymbol{N}\left(\boldsymbol{D}_{k} \boldsymbol{\beta}, \sigma^{2} \boldsymbol{I}_{n_{k}}\right)
$$

for each $k=1, \ldots, K$, where $\boldsymbol{I}_{n_{k}}$ denotes the $n_{k} \times n_{k}$ identity matrix. With adaptive sampling, the $k$ th group of observations is multivariate normal with the distribution specified for the final $n_{k}-n_{k-1}$ components of $\boldsymbol{X}_{k}$ in (7) conditionally on the number of observations, $n_{k}-n_{k-1}$, and their covariate values which appear in the final $n_{k}-n_{k-1}$ rows of $\boldsymbol{D}_{k}$.

The maximum likelihood estimates of $\boldsymbol{\beta}$ at successive analyses have the usual form

$$
\widehat{\boldsymbol{\beta}}_{k}=\left(\boldsymbol{D}_{k}^{T} \boldsymbol{D}_{k}\right)^{-1} \boldsymbol{D}_{k}^{T} \boldsymbol{X}_{k}, \quad k=1, \ldots, K .
$$

For each $k=1, \ldots, K$, we let

$$
\boldsymbol{V}_{k}=\left(\boldsymbol{D}_{k}^{T} \boldsymbol{D}_{k}\right)^{-1} \sigma^{2}
$$

and define the information for $\boldsymbol{c}^{T} \boldsymbol{\beta}$ at analysis $k$

$$
\mathcal{I}_{k}=\left(\boldsymbol{c}^{T} \boldsymbol{V}_{k} \boldsymbol{c}\right)^{-1}
$$

and the score statistic for $\boldsymbol{c}^{T} \boldsymbol{\beta}$

$$
S_{k}=\boldsymbol{c}^{T} \widehat{\boldsymbol{\beta}}_{k} \mathcal{I}_{k} .
$$

Let $\boldsymbol{A}$ be a $(p-1) \times p$ matrix of rank $p-1$ satisfying $\boldsymbol{A} \boldsymbol{c}=\mathbf{0}$ and define

$$
\boldsymbol{W}_{k}=\boldsymbol{A} \boldsymbol{V}_{k}^{-1} \widehat{\boldsymbol{\beta}}_{k} .
$$

In the non-adaptive setting we would have $\boldsymbol{C o v}\left(\boldsymbol{W}_{k}, \boldsymbol{c}^{T} \widehat{\boldsymbol{\beta}}\right)=\mathbf{0}$ so we can regard $\boldsymbol{W}_{k}$ as representing the information in $\widehat{\boldsymbol{\beta}}_{k}$ complementary to $\boldsymbol{c}^{T} \widehat{\boldsymbol{\beta}}_{k}$.

Jennison \& Turnbull (1997a) show that under non-adaptive sampling the joint distribution of the sequence of estimates $\widehat{\boldsymbol{\beta}}_{1}, \ldots, \widehat{\boldsymbol{\beta}}_{K}$ has a simple standard form and the sequence of score statistics for a linear combination of parameters can be embedded in a Brownian motion. Thus, $\left\{S_{k} ; k=1, \ldots, K\right\}$ defined above would be distributed as a Brownian motion with drift $\boldsymbol{c}^{T} \boldsymbol{\beta}$ observed at times $\left\{\mathcal{I}_{k} ; k=1, \ldots, K\right\}$. We now show that this property is retained under adaptive sampling schemes which allow the choice of observations in each group $k$ to depend on the previous estimate $\boldsymbol{c}^{T} \widehat{\boldsymbol{\beta}}_{k-1}$ but not on the orthogonal statistic $\boldsymbol{W}_{k-1}$.

\section{Theorem 2.}

Under the group sequential scheme described above suppose that for each $k=2, \ldots, K$ the number of observations in group $k$ and their covariate values depend on the previous design matrices $\boldsymbol{D}_{1}, \ldots, \boldsymbol{D}_{k-1}$ and observed score statistics $S_{1}, \ldots, S_{k-1}$ but not on $\boldsymbol{W}_{k-1}$. Then

$$
S_{1} \mid \mathcal{I}_{1} \sim N\left(\mathcal{I}_{1} \boldsymbol{c}^{T} \boldsymbol{\beta}, \mathcal{I}_{1}\right)
$$


and for each $k=2, \ldots, K$,

$$
S_{k}-S_{k-1} \mid \mathcal{I}_{1}, \ldots, \mathcal{I}_{k}, S_{1}, \ldots, S_{k-1} \sim N\left(\left\{\mathcal{I}_{k}-\mathcal{I}_{k-1}\right\} \boldsymbol{c}^{T} \boldsymbol{\beta}, \mathcal{I}_{k}-\mathcal{I}_{k-1}\right)
$$

\section{Proof of Theorem 2.}

The proof follows the same sequence of steps as in the proof of Theorem 1 , adapting these where necessary to deal with the general case. Let $G_{k}=\left\{\boldsymbol{D}_{1}, \ldots, \boldsymbol{D}_{k}\right\}, k=1, \ldots, K$, so $G_{k}$ also determines $\mathcal{I}_{1}, \ldots, \mathcal{I}_{k}$ and $G_{k}$ and $\boldsymbol{c}^{T} \widehat{\boldsymbol{\beta}}_{k}$ together determine $S_{k}$.

The proof is by induction with the inductive hypothesis

$$
\mathcal{H}_{k}:\left\{\begin{array}{l}
S_{k}-S_{k-1} \perp S_{1}, \ldots, S_{k-1} \mid G_{k} \\
S_{k}-S_{k-1} \mid G_{k} \sim N\left(\left\{\mathcal{I}_{k}-\mathcal{I}_{k-1}\right\} \boldsymbol{c}^{T} \boldsymbol{\beta}, \mathcal{I}_{k}-\mathcal{I}_{k-1}\right) \\
\boldsymbol{W}_{k} \perp S_{1}, \ldots, S_{k} \mid G_{k} \\
\boldsymbol{W}_{k} \mid G_{k} \sim \boldsymbol{N}\left(\boldsymbol{A} \boldsymbol{V}_{k}^{-1} \boldsymbol{\beta}, \boldsymbol{A} \boldsymbol{V}_{k}^{-1} \boldsymbol{A}^{T}\right)
\end{array}\right.
$$

where $S_{0}$ and $\mathcal{I}_{0}$ are defined to be zero. The theorem follows directly from $\mathcal{H}_{1}, \ldots, \mathcal{H}_{K}$.

The initial hypothesis $\mathcal{H}_{1}$ is easily checked. For $k=1, \ldots, K-1$, we prove $\mathcal{H}_{k+1}$ from $\mathcal{H}_{k}$ by establishing the following results:

1. $S_{k+1}-S_{k} \perp S_{1}, \ldots, S_{k} \mid G_{k+1}$ and

$$
S_{k+1}-S_{k} \mid G_{k+1} \sim N\left(\left\{\mathcal{I}_{k+1}-\mathcal{I}_{k}\right\} \boldsymbol{c}^{T} \boldsymbol{\beta}, \mathcal{I}_{k+1}-\mathcal{I}_{k}\right)
$$

2. $\boldsymbol{W}_{k+1} \perp S_{1}, \ldots, S_{k} \mid G_{k+1}$ and

$$
\boldsymbol{W}_{k+1} \mid G_{k+1} \sim \boldsymbol{N}\left(\boldsymbol{A} \boldsymbol{V}_{k+1}^{-1} \boldsymbol{\beta}, \boldsymbol{A} \boldsymbol{V}_{k+1}^{-1} \boldsymbol{A}^{T}\right)
$$

3. $\boldsymbol{W}_{k+1} \perp S_{k+1}-S_{k} \mid G_{k+1}, S_{1}, \ldots, S_{k}$.

Note that Results 2 and 3 imply $\boldsymbol{W}_{k+1} \perp S_{1}, \ldots, S_{k+1} \mid G_{k+1}$ as needed for $\mathcal{H}_{k+1}$. In proving these results, we use extensions of the properties of $\boldsymbol{W}_{k}$ stated in $\mathcal{H}_{k}$. Since $G_{k+1}$ comprises $G_{k}$ plus the number of observations in group $k+1$ and their covariate values, all of which depend only on $G_{k}$ and $S_{1}, \ldots, S_{k}$, it follows from $\mathcal{H}_{k}$ that also

$$
\boldsymbol{W}_{k} \perp S_{1}, \ldots, S_{k} \mid G_{k+1}
$$

and the conditional distribution of $\boldsymbol{W}_{k}$ given $G_{k+1}$ is the same as that given $G_{k}$, i.e.,

$$
\boldsymbol{W}_{k} \mid G_{k+1} \sim \boldsymbol{N}\left(\boldsymbol{A} \boldsymbol{V}_{k}^{-1} \boldsymbol{\beta}, \boldsymbol{A} \boldsymbol{V}_{k}^{-1} \boldsymbol{A}^{T}\right)
$$


Result 1.

We can decompose $S_{k+1}-S_{k}$ as

$$
\begin{aligned}
S_{k+1} & -S_{k}=\mathcal{I}_{k+1} \boldsymbol{c}^{T} \widehat{\boldsymbol{\beta}}_{k+1}-\mathcal{I}_{k} \boldsymbol{c}^{T} \widehat{\boldsymbol{\beta}}_{k} \\
& =\mathcal{I}_{k+1} \boldsymbol{c}^{T} \boldsymbol{V}_{k+1}\left(\boldsymbol{V}_{k+1}^{-1} \widehat{\boldsymbol{\beta}}_{k+1}-\boldsymbol{V}_{k}^{-1} \widehat{\boldsymbol{\beta}}_{k}\right)+\boldsymbol{c}^{T}\left(\mathcal{I}_{k+1} \boldsymbol{V}_{k+1} \boldsymbol{V}_{k}^{-1}-\mathcal{I}_{k} \boldsymbol{I}_{p}\right) \widehat{\boldsymbol{\beta}}_{k}
\end{aligned}
$$

Partitioning the data at analysis $k+1$ and the associated design matrix as

$$
\boldsymbol{X}_{k+1}=\left(\begin{array}{c}
\boldsymbol{X}_{k} \\
\boldsymbol{X}_{+}
\end{array}\right) \quad \text { and } \quad \boldsymbol{D}_{k+1}=\left(\begin{array}{c}
\boldsymbol{D}_{k} \\
\boldsymbol{D}_{+}
\end{array}\right)
$$

we see that

$$
\boldsymbol{V}_{k+1}^{-1} \widehat{\boldsymbol{\beta}}_{k+1}-\boldsymbol{V}_{k}^{-1} \widehat{\boldsymbol{\beta}}_{k}=\left(\boldsymbol{D}_{k+1}^{T} \boldsymbol{X}_{k+1}-\boldsymbol{D}_{k}^{T} \boldsymbol{X}_{k}\right) \sigma^{-2}=\boldsymbol{D}_{+}^{T} \boldsymbol{X}_{+} \sigma^{-2}
$$

The data $\boldsymbol{X}_{+}$in stage $k+1$ have conditional distribution given $G_{k+1}$

$$
\boldsymbol{X}_{+} \mid G_{k+1} \sim \boldsymbol{N}\left(\boldsymbol{D}_{+} \boldsymbol{\beta}, \boldsymbol{I}_{n_{k+1}-n_{k}} \sigma^{2}\right)
$$

so

$$
\boldsymbol{V}_{k+1}^{-1} \widehat{\boldsymbol{\beta}}_{k+1}-\boldsymbol{V}_{k}^{-1} \widehat{\boldsymbol{\beta}}_{k} \mid G_{k+1} \sim \boldsymbol{N}\left(\boldsymbol{D}_{+}^{T} \boldsymbol{D}_{+} \boldsymbol{\beta} \sigma^{-2}, \boldsymbol{D}_{+}^{T} \boldsymbol{D}_{+} \sigma^{-2}\right) .
$$

From the definitions of $\boldsymbol{V}_{k+1}$ and $\boldsymbol{V}_{k}$,

$$
\boldsymbol{D}_{+}^{T} \boldsymbol{D}_{+}=\boldsymbol{V}_{k+1}^{-1} \sigma^{2}-\boldsymbol{V}_{k}^{-1} \sigma^{2}
$$

so, conditionally on $G_{k+1}$,

$$
\boldsymbol{V}_{k+1}^{-1} \widehat{\boldsymbol{\beta}}_{k+1}-\boldsymbol{V}_{k}^{-1} \widehat{\boldsymbol{\beta}}_{k} \mid G_{k+1} \sim \boldsymbol{N}\left(\left\{\boldsymbol{V}_{k+1}^{-1}-\boldsymbol{V}_{k}^{-1}\right\} \boldsymbol{\beta}, \boldsymbol{V}_{k+1}^{-1}-\boldsymbol{V}_{k}^{-1}\right)
$$

and the first term in (10) is distributed, given $G_{k+1}$, as

$$
\begin{aligned}
& \mathcal{I}_{k+1} \boldsymbol{c}^{T} \boldsymbol{V}_{k+1}\left(\boldsymbol{V}_{k+1}^{-1} \widehat{\boldsymbol{\beta}}_{k+1}-\boldsymbol{V}_{k}^{-1} \widehat{\boldsymbol{\beta}}_{k}\right) \mid G_{k+1} \\
& \quad \sim N\left(\mathcal{I}_{k+1} \boldsymbol{c}^{T}\left\{\boldsymbol{I}_{p}-\boldsymbol{V}_{k+1} \boldsymbol{V}_{k}^{-1}\right\} \boldsymbol{\beta}, \mathcal{I}_{k+1}-\mathcal{I}_{k+1}^{2} \boldsymbol{c}^{T} \boldsymbol{V}_{k+1} \boldsymbol{V}_{k}^{-1} \boldsymbol{V}_{k+1} \boldsymbol{c}\right)
\end{aligned}
$$

This term depends only on data gathered in stage $k+1$ and, hence, it is conditionally independent, given $G_{k+1}$, of $S_{1}, \ldots, S_{k}$ and of the second term in (10) which is known at stage $k$.

Since $\boldsymbol{A}$ has rank $p-1$ and $\boldsymbol{A} \boldsymbol{c}=\mathbf{0}$, all $p$-vectors orthogonal to $\boldsymbol{c}$ can be written as multiples of $\boldsymbol{A}$. The definitions of $\mathcal{I}_{k}$ and $\mathcal{I}_{k+1}$ imply

$$
\boldsymbol{c}^{T}\left(\mathcal{I}_{k+1} \boldsymbol{V}_{k+1} \boldsymbol{V}_{k}^{-1}-\mathcal{I}_{k} \boldsymbol{I}_{p}\right) \boldsymbol{V}_{k} \boldsymbol{c}=0
$$

so

$$
\boldsymbol{c}^{T}\left(\mathcal{I}_{k+1} \boldsymbol{V}_{k+1} \boldsymbol{V}_{k}^{-1}-\mathcal{I}_{k} \boldsymbol{I}_{p}\right) \boldsymbol{V}_{k}=\boldsymbol{M A}
$$


for some $1 \times(p-1)$ row vector $\boldsymbol{M}$. Thus, the second term of (10) can be written as

$$
\boldsymbol{M A} \boldsymbol{V}_{k}^{-1} \widehat{\boldsymbol{\beta}}_{k}=\boldsymbol{M} \boldsymbol{W}_{k},
$$

where

$$
\boldsymbol{M A} \boldsymbol{V}_{k}^{-1}=\boldsymbol{c}^{T}\left(\mathcal{I}_{k+1} \boldsymbol{V}_{k+1} \boldsymbol{V}_{k}^{-1}-\mathcal{I}_{k} \boldsymbol{I}_{p}\right)
$$

By (8) and (9),

$$
M W_{k} \perp S_{1}, \ldots, S_{k} \mid G_{k+1}
$$

and

$$
\boldsymbol{M} \boldsymbol{W}_{k} \mid G_{k+1} \sim N\left(\boldsymbol{M} \boldsymbol{A} \boldsymbol{V}_{k}^{-1} \boldsymbol{\beta}, \boldsymbol{M} \boldsymbol{A} \boldsymbol{V}_{k}^{-1} \boldsymbol{A}^{T} \boldsymbol{M}^{T}\right) .
$$

Substituting for $\boldsymbol{M} \boldsymbol{A} \boldsymbol{V}_{k}^{-1}$ from (13) and simplifying we find that, conditionally on $G_{k+1}$, the second term in (10) is distributed as

$$
\begin{aligned}
& \boldsymbol{c}^{T}\left(\mathcal{I}_{k+1} \boldsymbol{V}_{k+1} \boldsymbol{V}_{k}^{-1}-\mathcal{I}_{k} \boldsymbol{I}_{p}\right) \widehat{\boldsymbol{\beta}}_{k} \mid G_{k+1} \\
& \quad \sim N\left(\mathcal{I}_{k+1} \boldsymbol{c}^{T} \boldsymbol{V}_{k+1} \boldsymbol{V}_{k}^{-1} \boldsymbol{\beta}-\mathcal{I}_{k} \boldsymbol{c}^{T} \boldsymbol{\beta}, \mathcal{I}_{k+1}^{2} \boldsymbol{c}^{T} \boldsymbol{V}_{k+1} \boldsymbol{V}_{k}^{-1} \boldsymbol{V}_{k+1} \boldsymbol{c}-\mathcal{I}_{k}\right) .
\end{aligned}
$$

From (12) and (14) and the conditional independence of the two terms in (10) given $G_{k+1}$, we obtain

$$
S_{k+1}-S_{k} \mid G_{k+1} \sim N\left(\left\{\mathcal{I}_{k+1}-\mathcal{I}_{k}\right\} \boldsymbol{c}^{T} \boldsymbol{\beta}, \mathcal{I}_{k+1}-\mathcal{I}_{k}\right) .
$$

With the conditional independence of each term in (10) and $S_{1} \ldots, S_{k}$ given $G_{k+1}$, our Result 1 follows.

Result 2.

We subdivide $\boldsymbol{W}_{k+1}$ into $\boldsymbol{W}_{k}$ and $\boldsymbol{W}_{k+1}-\boldsymbol{W}_{k}$. Now,

$$
\boldsymbol{W}_{k+1}-\boldsymbol{W}_{k}=\boldsymbol{A}\left(\boldsymbol{V}_{k+1}^{-1} \widehat{\boldsymbol{\beta}}_{k+1}-\boldsymbol{V}_{k}^{-1} \widehat{\boldsymbol{\beta}}_{k}\right)
$$

and it follows from properties of $\boldsymbol{V}_{k+1}^{-1} \widehat{\boldsymbol{\beta}}_{k+1}-\boldsymbol{V}_{k}^{-1} \widehat{\boldsymbol{\beta}}_{k}$ established in proving Result 1 that

$$
\begin{aligned}
\boldsymbol{W}_{k+1} & -\boldsymbol{W}_{k} \mid G_{k+1} \\
& \sim \boldsymbol{N}\left(\boldsymbol{A}\left\{\boldsymbol{V}_{k+1}^{-1}-\boldsymbol{V}_{k}^{-1}\right\} \boldsymbol{\beta}, \boldsymbol{A} \boldsymbol{V}_{k+1}^{-1} \boldsymbol{A}^{T}-\boldsymbol{A} \boldsymbol{V}_{k}^{-1} \boldsymbol{A}^{T}\right) .
\end{aligned}
$$

As $\boldsymbol{W}_{k+1}-\boldsymbol{W}_{k}$ is a function only of $\boldsymbol{X}_{+}$, the data gathered in stage $k+1$,

$$
\boldsymbol{W}_{k+1}-\boldsymbol{W}_{k} \perp S_{1}, \ldots, S_{k} \mid G_{k+1}
$$

which, with (8), implies

$$
\boldsymbol{W}_{k+1} \perp S_{1}, \ldots, S_{k} \mid G_{k+1} .
$$

Also,

$$
\boldsymbol{W}_{k+1}-\boldsymbol{W}_{k} \perp \boldsymbol{W}_{k} \mid G_{k+1}
$$


and combining (9), (15) and (16) gives our Result 2.

\section{Result 3.}

We can write $\boldsymbol{W}_{k+1}$ as

$$
\boldsymbol{W}_{k+1}=\left(\boldsymbol{W}_{k+1}-\boldsymbol{W}_{k}\right)+\boldsymbol{W}_{k}=\boldsymbol{A}\left(\boldsymbol{V}_{k+1}^{-1} \widehat{\boldsymbol{\beta}}_{k+1}-\boldsymbol{V}_{k}^{-1} \widehat{\boldsymbol{\beta}}_{k}\right)+\boldsymbol{W}_{k} .
$$

By (10) and the subsequent discussion,

$$
S_{k+1}-S_{k}=\mathcal{I}_{k+1} c^{T} \boldsymbol{V}_{k+1}\left(\boldsymbol{V}_{k+1}^{-1} \widehat{\boldsymbol{\beta}}_{k+1}-\boldsymbol{V}_{k}^{-1} \widehat{\boldsymbol{\beta}}_{k}\right)+\boldsymbol{M} \boldsymbol{W}_{k}
$$

where $\boldsymbol{M} \boldsymbol{A} \boldsymbol{V}_{k}^{-1}=\boldsymbol{c}^{T}\left(\mathcal{I}_{k+1} \boldsymbol{V}_{k+1} \boldsymbol{V}_{k}^{-1}-\mathcal{I}_{k} \boldsymbol{I}_{p}\right)$. Both these expressions involve new data from stage $k+1$ through $\boldsymbol{V}_{k+1}^{-1} \widehat{\boldsymbol{\beta}}_{k+1}-\boldsymbol{V}_{k}^{-1} \widehat{\boldsymbol{\beta}}_{k}$ as well as $\boldsymbol{W}_{k}$ which is known at stage $k$. Conditionally on $G_{k+1}$ and $S_{1}, \ldots, S_{k}$, the terms $\boldsymbol{V}_{k+1}^{-1} \widehat{\boldsymbol{\beta}}_{k+1}-\boldsymbol{V}_{k}^{-1} \widehat{\boldsymbol{\beta}}_{k}$ and $\boldsymbol{W}_{k}$ are independent with, from (11),

$$
\boldsymbol{V}_{k+1}^{-1} \widehat{\boldsymbol{\beta}}_{k+1}-\boldsymbol{V}_{k}^{-1} \widehat{\boldsymbol{\beta}}_{k} \sim \boldsymbol{N}\left(\left\{\boldsymbol{V}_{k+1}^{-1}-\boldsymbol{V}_{k}^{-1}\right\} \boldsymbol{\beta}, \boldsymbol{V}_{k+1}^{-1}-\boldsymbol{V}_{k}^{-1}\right)
$$

and, by $\mathcal{H}_{k}$

$$
\boldsymbol{W}_{k} \sim \boldsymbol{N}\left(\boldsymbol{A} \boldsymbol{V}_{k}^{-1} \boldsymbol{\beta}, \boldsymbol{A} \boldsymbol{V}_{k}^{-1} \boldsymbol{A}^{T}\right)
$$

Thus, conditionally, $\boldsymbol{W}_{k+1}$ and $S_{k+1}-S_{k}$ are multivariate normal with covariance matrix

$$
\begin{aligned}
& \boldsymbol{A}\left(\boldsymbol{V}_{k+1}^{-1}-\boldsymbol{V}_{k}^{-1}\right) \boldsymbol{V}_{k+1} \boldsymbol{c} \mathcal{I}_{k+1}+\boldsymbol{A} \boldsymbol{V}_{k}^{-1} \boldsymbol{A}^{T} \boldsymbol{M}^{T} \\
= & \boldsymbol{A}\left(\boldsymbol{V}_{k+1}^{-1}-\boldsymbol{V}_{k}^{-1}\right) \boldsymbol{V}_{k+1} \boldsymbol{c} \mathcal{I}_{k+1}+\boldsymbol{A}\left(\mathcal{I}_{k+1} \boldsymbol{V}_{k}^{-1} \boldsymbol{V}_{k+1}-\mathcal{I}_{k}\right) \boldsymbol{c} \\
= & \boldsymbol{A} \boldsymbol{c} \mathcal{I}_{k+1}-\boldsymbol{A} \boldsymbol{V}_{k}^{-1} \boldsymbol{V}_{k+1} \boldsymbol{c} \mathcal{I}_{k+1}+\boldsymbol{A} \boldsymbol{V}_{k}^{-1} \boldsymbol{V}_{k+1} \boldsymbol{c} \mathcal{I}_{k+1}-\boldsymbol{A} \boldsymbol{c} \mathcal{I}_{k} \\
= & \boldsymbol{A} \boldsymbol{c}\left(\mathcal{I}_{k+1}-\mathcal{I}_{k}\right)=\mathbf{0}
\end{aligned}
$$

since $\boldsymbol{A c}=\mathbf{0}$. Therefore

$$
\boldsymbol{W}_{k+1} \perp S_{k+1}-S_{k} \mid G_{k+1}, S_{1}, \ldots, S_{k}
$$

as required for our Result 3. This completes the proof.

Theorem 2 is easily extended to the case of correlated observations using the device employed by Jennison \& Turnbull (1997b) in proving Case 2 of their Theorem 2. Define transformed variables

$$
Z_{i}=\left\{\operatorname{Var}\left(X_{i}\right)-\sum_{j=1}^{i-1} \lambda_{i j}^{2}\right\}^{-1 / 2}\left(X_{i}-\sum_{j=1}^{i-1} \lambda_{i j} Z_{j}\right), \quad i=1,2, \ldots,
$$

where $\lambda_{i j}$ is the covariance that would arise between $X_{i}$ and $Z_{j}$ if the same sequence of observations were made under a non-adaptive sampling rule. Each new $Z_{i}$ is now 
conditionally independent of $Z_{1}, \ldots, Z_{i-1}$, given the covariate values for the first $i$ observations. Thus, Theorem 2 can be applied to deduce properties of score statistics obtained from the transformed data. Because the same maximum likelihood estimates $\widehat{\boldsymbol{\beta}}_{k}, k=1, \ldots, K$, arise from both the original and transformed data sequences, the same distributional result must also apply to the score statistic sequence for the untransformed data.

\section{Examples}

We illustrate the application of data-dependent sampling in the comparison of two normal means described in Section 2.1. Using the notation of that section, suppose we wish to test the null hypothesis $H_{0}: \theta=0$ against the one-sided alternative $\theta>0$ with Type I error probability $\alpha$ and power $1-\beta$ at $\theta=\delta$, in a group sequential study with up to $K$ analyses. A family of group sequential one-sided tests has been proposed by Emerson \& Fleming (1989) for the case $\alpha=\beta$ and extended by Pampallona \& Tsiatis (1994) to allow unequal Type I and II error probabilities. The tests are designed on the assumption that information levels $\mathcal{I}_{\theta 1}, \ldots, \mathcal{I}_{\theta K}$ are equally spaced. Jennison \& Turnbull (2000, Sec. 4.2) refer to this family as the "power family" of one-sided tests and describe the tests in terms of stopping boundaries for the $Z$-statistics

$$
Z_{k}=S_{\theta k} / \sqrt{ } \mathcal{I}_{\theta k}, \quad k=1, \ldots, K
$$

The family of tests is indexed by a parameter $\Delta$ and the test with parameter $\Delta$ stops to accept $H_{0}$ if $Z_{k} \leq a_{k}$ or to reject $H_{0}$ if $Z_{k} \geq b_{k}$ at each stage $k=1, \ldots, K$, where

$$
a_{k}=\delta \sqrt{ } \mathcal{I}_{\theta k}-\tilde{C}_{2}(k / K)^{\Delta-1 / 2}, \quad b_{k}=\tilde{C}_{1}(k / K)^{\Delta-1 / 2}
$$

and the final information level satisfies

$$
\mathcal{I}_{\theta K}=\frac{\left\{\tilde{C}_{1}+\tilde{C}_{2}\right\}^{2}}{\delta^{2}}
$$

ensuring the upper and lower boundaries meet at analysis $K$. Values of the constants $\tilde{C}_{1}$ and $\tilde{C}_{2}$ needed to achieve particular error rates $\alpha$ and $\beta$ for a variety of combinations of $K$ and $\Delta$ are tabulated by Jennison \& Turnbull (2000, Tables 4.1 to 4.3).

An adaptive sampling rule can be used to vary the proportions of observations on treatments $A$ and $B$ as the study progresses, as long as the total number of observations in each stage is adjusted so that the planned, equally spaced information levels $\mathcal{I}_{\theta k}=(k / K)\left\{\tilde{C}_{1}+\tilde{C}_{2}\right\}^{2} / \delta^{2}, k=1, \ldots, K$, are still attained. Then, Theorem 1 implies that $S_{\theta 1}, \ldots, S_{\theta K}$, and hence $Z_{1}, \ldots, Z_{K}$, have their usual joint distributions and error probabilities will equal $\alpha$ and $\beta$ as required. If, instead, the total number of observations in stage $k$ is fixed but the proportions allocated to the two treatments are 
chosen adaptively, the information increment $\mathcal{I}_{\theta k}-\mathcal{I}_{\theta, k-1}$ decreases as the sampling rule departs from equal allocation and, as well as reducing the total information observed, the adaptive sampling rule will create a dependency of $\mathcal{I}_{\theta k}$ on $S_{k-1}$.

The "error spending" approach of Lan \& DeMets (1983) is a popular choice when it is difficult to control variation in group sizes due to random factors in patient accrual and data collection. It should be stressed that the methods used to compute error rates of error spending tests rely on the assumption that each new information level $\mathcal{I}_{\theta k}$ is conditionally independent of previous test statistics $S_{\theta 1}, \ldots, S_{\theta, k-1}$ or $Z_{1}, \ldots, Z_{k-1}$ given $\mathcal{I}_{\theta 1}, \ldots, \mathcal{I}_{\theta, k-1}$ and this property can easily be lost under adaptive sampling. One could insist that a pre-specified information level be achieved at each analysis but this would negate the value of the error spending approach in handling unequal and unpredictable information increments. A more flexible option is, at each stage, to set the date of the next analysis to meet a pre-specified target information level under the chosen treatment allocation rule and assuming average recruitment and data collection times. Since variation of the attained information level about this target will be unrelated to previous observations, the assumption noted above will hold and an error spending test can be implemented in the usual way.

One way to create an adaptive sampling rule which reduces the number of subjects assigned to the inferior treatment arm is through a loss function defined in terms of the numbers of subjects allocated to each treatment arm with a lower cost assigned to those on the better treatment. Let $N_{A}$ and $N_{B}$ denote the total sample sizes on treatments $A$ and $B$ respectively when the test terminates. Hayre (1979) proposed a loss function of the form

$$
L(\theta)=u(\theta) N_{A}+v(\theta) N_{B} .
$$

Supposing a high response is desirable, the inferior treatment is $A$ if $\theta<0$ and $B$ if $\theta>0$ and the weights $u(\theta)$ and $v(\theta)$ should reflect this, $v(\theta)$ increasing in $\theta$ for $\theta>0$ and $u(\theta)$ increasing as $\theta$ decreases for $\theta<0$. The weighted combination of ITN and ASN used by Louis (1975) can be expressed in this form; the same type of loss function was used by Hayre \& Turnbull (1981a) in tests for survival and binomial data and by Hayre \& Turnbull $(1981 b, c)$ in applications to point and interval estimation. Jennison \& Turnbull (2000, Sec. 17.1) consider the particular case

$$
L(\theta)= \begin{cases}N_{A}+a^{\theta / \delta} N_{B} & \text { for } \theta \geq 0, \\ a^{-\theta / \delta} N_{A}+N_{B} & \text { for } \theta \leq 0,\end{cases}
$$

where $a$ is a chosen constant and $\delta$ the value of $\theta$ at which the power condition is set.

Let $\mathcal{I}_{\theta T}$ denote the information for $\theta$ on termination. The allocation ratio minimizing (18) subject to

$$
\frac{N_{A} N_{B}}{N_{A}+N_{B}}=\mathcal{I}_{\theta T}
$$


is

$$
\frac{N_{A}}{N_{B}}=w(\theta)
$$

where

$$
w(\theta)=\left\{\frac{v(\theta)}{u(\theta)}\right\}^{1 / 2} .
$$

Although $\theta$ is unknown, successive estimates can be substituted in this optimal ratio to create the following adaptive rule.

\section{Adaptive Sampling Rule}

At the first stage, set

$$
n_{A 1}=\{1+w(\tilde{\theta})\} \mathcal{I}_{\theta 1} \quad \text { and } \quad n_{B 1}=\frac{1+w(\tilde{\theta})}{w(\tilde{\theta})} \mathcal{I}_{\theta 1}
$$

where $\tilde{\theta}$ is a preliminary estimate of $\theta$ or, if preferred, simply use $w(\tilde{\theta})=1$. (Of course, these allocations must be rounded to integer values.)

In planning each stage $k=2, \ldots, K$, we aim for cumulative sample sizes in the ratio

$$
\frac{n_{A k}}{n_{B k}}=w\left(\hat{\theta}_{k-1}\right)
$$

subject to reaching the pre-specified information level $\mathcal{I}_{\theta k}$. This leads to group sizes

$$
\left\{1+w\left(\hat{\theta}_{k-1}\right)\right\} \mathcal{I}_{k}-n_{A, k-1} \quad \text { and } \quad \frac{1+w\left(\hat{\theta}_{k-1}\right)}{w\left(\hat{\theta}_{k-1}\right)} \mathcal{I}_{k}-n_{B, k-1}
$$

on treatments $A$ and $B$ respectively, again rounded to integers. If either of these group sizes is negative, that allocation is set to zero and sufficient observations taken on the other arm to achieve the required information level $\mathcal{I}_{\theta k}$. Alternatively, a minimum sample size per treatment arm may be specified at each stage and if either group size in (19) falls below this, the minimum group size is used in its place and the sample size on the other arm chosen to achieve $\mathcal{I}_{\theta k}$.

In applications of this adaptive sampling rule reported by Jennison \& Turnbull (2000, Sec. 17.1), tests have Type I error probability $\alpha=0.05$, power $1-\beta=0.9$ at $\theta=\delta$, and boundary values given by (17) with constants $\tilde{C}_{1}$ and $\tilde{C}_{2}$ for $\Delta=0$ obtained from their Table 4.2. Equal group sizes, $n_{A 1}=n_{B 1}$, are used initially, after which the adaptive allocation rule (19) is employed with the loss function (18), for which

$$
w(\theta)=a^{\theta /(2 \delta)} .
$$


Table 1: Properties of power family one-sided tests with $\Delta=0$ and 5 groups of observations. Tests have Type I error probability $\alpha=0.05$ at $\theta=0$, power $1-\beta=0.9$ at $\theta=\delta$ and use the adaptive allocation rule (19) with $w(\theta)=a^{\theta /(2 \delta)}$. Expected sample sizes on treatments $A$ and $B$ are expressed as percentages of the sample size per treatment arm in a fixed sample test with equal treatment allocation and the same error probabilities.

\begin{tabular}{|c|c|c|c|c|c|c|c|}
\hline \multirow[b]{3}{*}{$\theta$} & \multirow{3}{*}{$\begin{array}{l}\text { Equal sampling } \\
E\left(N_{A}\right)=E\left(N_{B}\right)\end{array}$} & \multicolumn{6}{|c|}{ Adaptive sampling, $a=1.5$} \\
\hline & & & & \multicolumn{2}{|c|}{$\%$ Reduction in ITN * } & \multicolumn{2}{|c|}{$\%$ Increase in ASN $†$} \\
\hline & & $E\left(N_{A}\right)$ & $E\left(N_{B}\right)$ & Target & Actual & Target & Actual \\
\hline$-\delta$ & 37.3 & 35.8 & 39.1 & 9.2 & 4.0 & 1.0 & 0.4 \\
\hline$-\delta / 2$ & 47.0 & 46.2 & 48.3 & 4.8 & 1.7 & 0.3 & 0.5 \\
\hline 0 & 63.8 & 65.0 & 62.9 & - & - & 0.0 & 0.2 \\
\hline$\delta / 2$ & 80.1 & 84.8 & 76.2 & 4.8 & 4.9 & 0.3 & 0.5 \\
\hline$\delta^{\prime}$ & 71.8 & 79.0 & 65.9 & 9.2 & 8.2 & 1.0 & 0.9 \\
\hline $3 \delta / 2$ & 54.3 & 62.9 & 48.1 & 13.1 & 11.4 & 2.3 & 2.2 \\
\hline \multirow[t]{3}{*}{$2 \delta$} & 43.7 & 52.5 & 37.7 & 16.7 & 13.7 & 4.2 & 3.2 \\
\hline & Equal sampling & \multicolumn{6}{|c|}{ Adaptive sampling, $a=2$} \\
\hline & & & & \multicolumn{2}{|c|}{$\%$ Reduction in ITN } & \multicolumn{2}{|c|}{$\%$ Increase in $\mathrm{ASN}$} \\
\hline$\theta$ & $E\left(N_{A}\right)=E\left(N_{B}\right)$ & $E\left(N_{A}\right)$ & $E\left(N_{B}\right)$ & Target & Actual & Target & Actual \\
\hline$-\delta$ & 37.3 & 35.0 & 40.6 & 14.6 & 6.2 & 3.0 & 1.3 \\
\hline$-\delta / 2$ & 47.0 & 45.6 & 49.3 & 8.0 & 3.0 & 0.8 & 1.0 \\
\hline 0 & 63.8 & 66.0 & 62.5 & - & - & 0.0 & 0.7 \\
\hline$\delta / 2$ & 80.1 & 88.5 & 73.7 & 8.0 & 8.0 & 0.8 & 1.2 \\
\hline$\delta$ & 71.8 & 85.5 & 62.6 & 14.6 & 12.8 & 3.0 & 3.1 \\
\hline $3 \delta / 2$ & 54.3 & 71.0 & 44.8 & 20.3 & 17.5 & 6.9 & 6.6 \\
\hline \multirow[t]{3}{*}{$2 \delta^{\prime}$} & 43.7 & 61.3 & 34.7 & 25.0 & 20.6 & 12.5 & 9.8 \\
\hline & Equal sampling & \multicolumn{6}{|c|}{ Adaptive sampling, $a=4$} \\
\hline & & & & \multicolumn{2}{|c|}{$\%$ Reduction in ITN } & \multicolumn{2}{|c|}{$\%$ Increase in ASN } \\
\hline$\theta$ & $E\left(N_{A}\right)=E\left(N_{B}\right)$ & $E\left(N_{A}\right)$ & $E\left(N_{B}\right)$ & Target & Actual & Target & Actual \\
\hline$-\delta$ & 37.3 & 33.3 & 45.2 & 25.0 & 10.7 & 12.5 & 5.2 \\
\hline$-\delta / 2$ & 47.0 & 45.0 & 52.7 & 14.6 & 4.3 & 3.0 & 3.9 \\
\hline 0 & 63.8 & 69.3 & 62.1 & - & - & 0.0 & 3.0 \\
\hline$\delta / 2$ & 80.1 & 100.2 & 68.9 & 14.6 & 14.0 & 3.0 & 5.6 \\
\hline$\delta$ & 71.8 & 106.6 & 56.1 & 25.0 & 21.9 & 12.5 & 13.3 \\
\hline $3 \delta / 2$ & 54.3 & 101.0 & 38.9 & 32.3 & 28.4 & 29.5 & 28.8 \\
\hline $2 \delta$ & 43.7 & 96.9 & 29.9 & 37.5 & 31.6 & 56.2 & 45.1 \\
\hline
\end{tabular}

*ITN or "inferior treatment number" is the expected number of subjects on the inferior treatment $\dagger$ ASN or "average sample size" is the expected total number of subjects 
Table 2: Properties of power family one-sided tests with $\Delta=0$ and 10 groups of observations. Tests have Type I error probability $\alpha=0.05$ at $\theta=0$, power $1-\beta=0.9$ at $\theta=\delta$ and use the adaptive allocation rule (19) with $w(\theta)=a^{\theta /(2 \delta)}$. Expected sample sizes on treatments $A$ and $B$ are expressed as percentages of the sample size per treatment arm in a fixed sample test with equal treatment allocation and the same error probabilities.

\begin{tabular}{|c|c|c|c|c|c|c|c|}
\hline \multirow[b]{3}{*}{$\theta$} & \multirow{3}{*}{$\begin{array}{l}\text { Equal sampling } \\
E\left(N_{A}\right)=E\left(N_{B}\right)\end{array}$} & \multicolumn{6}{|c|}{ Adaptive sampling, $a=1.5$} \\
\hline & & & & \multicolumn{2}{|c|}{$\%$ Reduction in ITN * } & \multicolumn{2}{|c|}{$\%$ Increase in ASN $†$} \\
\hline & & $E\left(N_{A}\right)$ & $E\left(N_{B}\right)$ & Target & Actual & Target & Actual \\
\hline$-\delta$ & 33.4 & 30.9 & 36.6 & 9.2 & 7.5 & 1.0 & 1.0 \\
\hline$-\delta / 2$ & 43.0 & 41.7 & 44.7 & 4.8 & 3.0 & 0.3 & 0.5 \\
\hline 0 & 60.1 & 61.0 & 59.5 & - & - & 0.0 & 0.2 \\
\hline$\delta / 2$ & 77.0 & 81.3 & 73.1 & 4.8 & 5.1 & 0.3 & 0.3 \\
\hline$\delta^{\prime}$ & 68.2 & 75.3 & 62.7 & 9.2 & 8.1 & 1.0 & 1.2 \\
\hline $3 \delta / 2$ & 50.2 & 58.5 & 44.4 & 13.1 & 11.6 & 2.3 & 2.5 \\
\hline \multirow[t]{3}{*}{$2 \delta$} & 38.9 & 48.0 & 32.9 & 16.7 & 15.4 & 4.2 & 4.0 \\
\hline & Equal sampling & \multicolumn{6}{|c|}{ Adaptive sampling, $a=2$} \\
\hline & & & & \multicolumn{2}{|c|}{ \% Reduction in ITN } & \multicolumn{2}{|c|}{$\%$ Increase in ASN } \\
\hline$\theta$ & $E\left(N_{A}\right)=E\left(N_{B}\right)$ & $E\left(N_{A}\right)$ & $E\left(N_{B}\right)$ & Target & Actual & Target & Actual \\
\hline$-\delta$ & 33.4 & 29.6 & 39.7 & 14.6 & 11.4 & 3.0 & 3.7 \\
\hline$-\delta / 2$ & 43.0 & 41.0 & 46.2 & 8.0 & 4.7 & 0.8 & 1.4 \\
\hline 0 & 60.1 & 61.8 & 59.2 & - & - & 0.0 & 0.7 \\
\hline$\delta / 2$ & 77.0 & 84.9 & 70.9 & 8.0 & 7.9 & 0.8 & 1.2 \\
\hline$\delta$ & 68.2 & 81.3 & 59.3 & 14.6 & 13.0 & 3.0 & 3.1 \\
\hline $3 \delta / 2$ & 50.2 & 66.1 & 41.0 & 20.3 & 18.3 & 6.9 & 6.7 \\
\hline \multirow[t]{3}{*}{$2 \delta$} & 38.9 & 57.5 & 29.9 & 25.0 & 23.1 & 12.5 & 12.3 \\
\hline & Equal sampling & \multicolumn{6}{|c|}{ Adaptive sampling, $a=4$} \\
\hline & & & & \multicolumn{2}{|c|}{$\%$ Reduction in ITN } & \multicolumn{2}{|c|}{$\%$ Increase in ASN } \\
\hline$\theta$ & $E\left(N_{A}\right)=E\left(N_{B}\right)$ & $E\left(N_{A}\right)$ & $E\left(N_{B}\right)$ & Target & Actual & Target & Actual \\
\hline$-\delta$ & 33.4 & 27.2 & 51.5 & 25.0 & 18.6 & 12.5 & 17.8 \\
\hline$-\delta / 2$ & 43.0 & 39.9 & 52.1 & 14.6 & 7.2 & 3.0 & 7.0 \\
\hline 0 & 60.1 & 64.5 & 59.5 & - & - & 0.0 & 3.2 \\
\hline$\delta / 2$ & 77.0 & 95.9 & 66.3 & 14.6 & 13.9 & 3.0 & 5.3 \\
\hline$\delta$ & 68.2 & 102.0 & 53.0 & 25.0 & 22.3 & 12.5 & 13.6 \\
\hline $3 \delta / 2$ & 50.2 & 97.3 & 35.5 & 32.3 & 29.3 & 29.5 & 32.3 \\
\hline $2 \delta$ & 38.9 & 101.9 & 25.2 & 37.5 & 35.2 & 56.2 & 63.4 \\
\hline
\end{tabular}

*ITN or "inferior treatment number" is the expected number of subjects on the inferior treatment $\dagger$ ASN or "average sample size" is the expected total number of subjects 
Expected sample sizes for these tests are proportional to $\sigma^{2} / \delta^{2}$ and it is convenient to express them as percentages of the sample size of per treatment arm required by a nonsequential test,

$$
\left\{\Phi^{-1}(1-\alpha)+\Phi^{-1}(1-\beta)\right\}^{2} 2 \sigma^{2} / \delta^{2}
$$

where $\Phi$ denotes the standard normal cumulative distribution function.

Properties of these adaptive group sequential tests are shown in Table 1 for designs with 5 groups of observations and in Table 2 for designs with 10 groups. Estimates of expected sample sizes are based on 250,000 simulations and have standard errors of less than 0.1. The "target" ITNs and ASNs are calculated using the optimal allocation ratio, $w(\theta)$, throughout the study. Since $\theta$ is unknown to the experimenter this rule is not a practical option, but the close agreement in many instances between target and actual values shows the effectiveness of the adaptive sampling rule in producing a sampling ratio close to $w(\theta)$ by the time the study terminates. When $\theta$ is equal to $-\delta$ or $-\delta / 2$, treatment $A$ is the inferior treatment and has the lower expected sample size; the roles of $A$ and $B$ are reversed for positive values of $\theta$. The stopping boundary (17) is not symmetric about $Z_{k}=0$, which explains the lack of equality between $E\left(N_{A}\right)$ and $E\left(N_{B}\right)$ when $\theta=0$ and the lack of a well-defined ITN in this case.

In these one-sided tests there is particular interest in expected sample sizes under $\theta=\delta$, the alternative at which the power condition has been set. For both 5 and 10 group designs, the choices $a=1.5, a=2$ and $a=4$ reduce the ITN by $8 \%, 13 \%$ and $22 \%$, respectively, with accompanying increases in $\mathrm{ASN}$ of around $1 \%, 3 \%$ and $13.5 \%$. Peto (1985) stresses the importance of a low total sample size since usually this is closely related to study duration - and a study must terminate before the benefits of a new treatment can be made available to the general patient population. Our results for $a=1.5$ and $a=2$ show that useful reductions in ITN can be gained with only minor increases in ASN, thereby meeting the requirements of both internal and external patients.

Calculations in the idealized situation, where the allocation ratio $w(\theta)$ is used throughout, indicate the best possible combinations of ITN and ASN. The group sequential design fixes the expected information on termination, $E\left(\mathcal{I}_{\theta T}\right)$. Since

$$
E\left(\mathcal{I}_{\theta T}\right)=E_{\theta}\left(\frac{N_{A} N_{B}}{N_{A}+N_{B}}\right)
$$

equal sampling of both treatments yields

$$
E_{\theta}\left(N_{A}\right)=E_{\theta}\left(N_{B}\right)=2 E\left(\mathcal{I}_{\theta T}\right)
$$

If treatment arms are sampled in a fixed ratio so that at each stage $k=1, \ldots, K$

$$
n_{A k} / n_{B k}=1+\gamma,
$$

then

$$
E\left(\mathcal{I}_{\theta T}\right)=E_{\theta}\left(\frac{N_{A} N_{B}}{N_{A}+N_{B}}\right)=E_{\theta}\left\{\frac{N_{A}}{\left(N_{A} / N_{B}\right)+1}\right\}=E_{\theta}\left(\frac{N_{A}}{2+\gamma}\right)
$$


and, hence,

$$
E_{\theta}\left(N_{A}\right)=\left(1+\frac{\gamma}{2}\right) 2 E\left(\mathcal{I}_{\theta T}\right)
$$

Similarly, we find

$$
E_{\theta}\left(N_{B}\right)=\left\{1-\frac{\gamma}{2(1+\gamma)}\right\} 2 E\left(\mathcal{I}_{\theta T}\right) .
$$

Substituting $x=\gamma /\{2(1+\gamma)\}$ for the case $\theta>0$, a little algebra shows that when the ITN is reduced to $(1-x)$ times its value under equal sampling, the expected sample size on the other treatment arm increases by a factor $\{1+x /(1-2 x)\}$ and the ASN increases by a factor of $\left\{1+x^{2} /(1-2 x)\right\}$. Note that for small $x$ the fractional increase in ASN is approximately the square of the fractional decrease in ITN, which explains why initial reductions in ITN, using $a=1.5$ or $a=2$ in (18), are attained with virtually no increase in ASN. At the other extreme, Robbins \& Siegmund (1974) note that, as a consequence of (20), the ITN can be reduced by at most $50 \%$ of its value under equal sampling and this only in the limit as the expected sample size for the other treatment arm approaches infinity.

Alternative expressions when $1+\gamma=w(\theta)=a^{\theta /(2 \delta)}$ are

$$
E_{\theta}\left(N_{A}\right)=\frac{1+a^{\theta /(2 \delta)}}{2} 2 E\left(\mathcal{I}_{\theta T}\right), \quad E_{\theta}\left(N_{B}\right)=\frac{1+a^{\theta /(2 \delta)}}{2 a^{\theta /(2 \delta)}} 2 E\left(\mathcal{I}_{\theta T}\right)
$$

and

$$
E_{\theta}\left(N_{A}\right)+E_{\theta}\left(N_{B}\right)=\frac{\left(1+a^{\theta /(2 \delta)}\right)^{2}}{4 a^{\theta /(2 \delta)}} 4 E\left(\mathcal{I}_{\theta T}\right) .
$$

Although these target values can only be attained approximately without knowledge of the true $\theta$, results in Tables 1 and 2 show that the proposed adaptive sampling rule produces expected sample sizes close to these for many combinations of $\theta$ and $\delta$.

\section{Discussion}

We have presented a strategy for data dependent treatment assignment that is easily incorporated in standard forms of group sequential test. Our proposed adaptive sampling rule can reduce the expected number of subjects allocated to the inferior treatment while only slightly increasing the expected total sample size. This avoids Peto's (1985) criticism that adaptive sampling disadvantages the wider patient population by delaying the study's outcome. In the ECMO studies mentioned in Section 1, clinicians were reluctant to randomize subjects to the control treatment since the evidence available before each study, while not conclusive, did lend support to the experimental therapy. Use of adaptive treatment assignment overcame the problem in this case: the argument that adaptive sampling rules offer subjects a greater chance of randomization to the superior treatment may be made more generally to persuade clinicians to enroll patients in a randomized 
study. If this argument is successful, the more rapid patient accrual will actually speed up the study, thereby benefiting the wider population.

Two requirements arise in establishing the distribution theory in Theorems 1 and 2 for test statistics from adaptive sequential designs and in using these results to construct valid group sequential tests. The first is that decisions on treatment allocation should depend on the estimated parameter of interest and not on other orthogonal statistics. This requirement is simple to accommodate as it is natural to define adaptive sampling rules in terms of such parameter estimates. The second condition is that observed information levels should not be influenced by the values of previous test statistics. This condition will be met if, at each stage, the length of time until the next analysis is adjusted in view of the current sampling allocation ratio so as to attain a pre-specified information level at that analysis. In many of the examples presented in Tables 1 and 2 the effect of adaptive sampling is to increase the average total sample size by only a few percent over that for equal sampling and in these cases such adjustments will be very minor. Often, information levels at successive analyses are unpredictable due to random variation about anticipated group sizes and the error spending approach of Lan \& DeMets (1983) is commonly used to handle this problem. When incorporating an adaptive sampling rule in an error spending test, it is sufficient to set calendar times of future analyses to attain target information levels assuming average patient recruitment, etc. Then, any further variation in the observed information level will not depend on previous observed data and the usual error spending computations are valid. Use of a maximum information design (see, for example, Jennison \& Turnbull, 2000, Sec. 7.2.2) is advisable to ensure the trial continues long enough to attain adequate power but complete independence of future increments in information from previous test statistics appears to be less crucial: Proschan, Follmann \& Waclawiw (1992) present convincing evidence that allowing current test statistics to influence future information levels has only minor effects on the error rates attained by error spending tests. Indeed, some authors have proposed making deliberate changes in analysis times in response to observed test statistics; see, for example, Betensky (1998).

Other drawbacks of adaptive sampling that have been cited include the apparent need for a rapid response from each subject and sensitivity of the resulting tests to time trends in the patient population. The formulae (19) for stage $k$ group sizes can be applied using the estimate $\hat{\theta}_{k-1}$ based on whatever data are available at analysis $k-1$, so all one really needs is sufficient information for $\theta$ to guide sampling proportions close to their target values by the time patient accrual ends. This may be unrealistic for survival studies in which follow-up extends well beyond the accrual period but it will be realistic in many other situations. The theory for normal linear models in Section 2.2 is useful in that it allows the modeling of time trends by, say, low order polynomials. Alternatively, arbitrary time trends can be dealt with by additive stage effects (see Jennison \& Turnbull, 2000, Sec. 17.2).

In conclusion, recent developments in group sequential procedures and theory for 
adaptive sampling rules provide the basis for incorporating data dependent treatment assignment in randomized clinical trials. In view of the renewed interest in adaptive

sampling exemplified by Tamura et al. (1994) and Andersen (1996), this methodology has an important role.

\section{$5 \quad$ References}

Anscombe, F.J. (1963). Sequential medical trials. J. Amer. Statist. Assoc., 58, 365-384.

Andersen, J.S. (1996). Clinical trial designs - made to order. J. Biopharm. Statist., 6, $515-522$.

Armitage, P. (1985). The search for optimality in clinical trials. Intern. Statist. Rev., 53, $15-24$.

Bather, J.A. (1985). On the allocation of treatments in sequential medical trials. Intern. Statist. Rev., 53, 1-13.

Berry, D.A. \& Fristedt, B. (1985). Bandit Problems: Sequential Allocation of Experiments, Chapman \& Hall, London.

Betensky, R.A. (1998). Construction of a continuous stopping boundary from an alpha spending function. Biometrics, 54, 1061-1071.

Colton, T. (1963). A model for selecting one of two medical treatments. J. Amer. Statist. Assoc., 58, 388-401.

Cornell, R.G., Landenberger, B.D. and Bartlett, R.H. (1986). Randomized play-thewinner clinical trials. Commun. Statist. A., 15, 159-178.

Emerson, S.S. and Fleming, T.R. (1989). Symmetric group sequential designs. Biometrics, 45, 905-923.

Hayre, L.S. (1979). Two-population sequential tests with three hypotheses. Biometrika, 66, 465-74.

Hayre, L.S. and Turnbull, B.W. (1981a). A class of simple approximate sequential tests for adaptive comparison of two treatments. Commun. Statist. A., 10, 2339-2360.

Hayre, L.S. and Turnbull, B.W. (1981b). Sequential estimation in two-armed exponential clinical trials. Biometrika, 68, 411-416.

Hayre, L.S. and Turnbull, B.W. (1981c). Estimation of the odds ratio in the two-armed bandit problem. Biometrika, 68, 661-668. 
Jennison, C. \& Turnbull, B. W. (1997a) Group sequential analysis incorporating covariate information. J. Amer. Statist. Assoc., 92, 1330-1341.

Jennison, C. and Turnbull, B.W. (1997b). Distribution theory of group sequential $t, \chi^{2}$ and $F$ tests for general linear models. Sequential Analysis, 16, 295-317.

Jennison, C. \& Turnbull, B.W. (2000). Group Sequential Methods with Applications to Clinical Trials, Chapman \& Hall/CRC, Boca Raton.

Lan, K.K.G. and DeMets, D.L. (1983). Discrete sequential boundaries for clinical trials. Biometrika, 70, 659-663.

Louis, T.A. (1975). Optimal allocation in sequential tests comparing the means of two Gaussian populations. Biometrika, 62, 359-369.

Pampallona, S. and Tsiatis, A.A. (1994). Group sequential designs for one-sided and two-sided hypothesis testing with provision for early stopping in favor of the null hypothesis. J. Statist. Planning and Inference, 42, 19-35.

Peto, R. (1985). Discussion of papers by J.A. Bather and P. Armitage. Intern. Statist. Rev., 53, 31-34,

Proschan, M.A., Follmann, D.A. and Waclawiw, M.A. (1992). Effect of assumption violations on Type I error rate in group sequential monitoring. Biometrics, 48, $1131-1143$.

Robbins, H. (1952). Some aspects of the sequential design of experiments. Bull. Amer. Math. Soc., 58, 527-535.

Robbins, H. and Siegmund, D.O. (1974). Sequential tests involving two populations. $J$. Amer. Statist. Assoc., 69, 132-139.

Tamura, R.N., Faries, D.E., Andersen, J.S. and Heiligenstein, J.H. (1994). A case study of an adaptive clinical trial in the treatment of out-patients with depressive disorder. J. Amer. Statist. Assoc., 89, 768-776.

Thompson, W.R. (1933) On the likelihood that one unknown probability exceeds another in view of the evidence of two samples. Biometrika, 25, 285-294.

Ware, J.H. (1989). Investigating therapies of potentially great benefit: ECMO (with discussion). Statistical Science, 4, 298-340. 